\title{
Anaesthetic management of a patient with Leigh's syndrome with central hypoventilation and obstructive sleep apnoea
}

Aileen Ling Wan $\underline{T a n}^{1}$, mBBs, Raymond Goy $^{1}$, mBBs, MMed, FAnzCA

\begin{abstract}
Leigh's syndrome, which is characterised by progressive neurodegeneration involving the brainstem and basal ganglia, belongs to a family of disorders classified as mitochondrial myopathies. It is most commonly transmitted by an autosomal recessive mode of inheritance, but can sometimes occur in a mitochondrial pattern. It typically presents during infancy with developmental delay and deterioration of brainstem function. Respiratory failure is the common cause of death and postoperative morbidity in patients with Leigh's disease. Herein, we report the case of a 17-year-old female patient with Leigh's syndrome who underwent general anaesthesia for a tracheostomy, which was performed in view of the patient's requirement for long-term ventilation and frequent toileting for secretions. Her respiratory complications included central hypoventilation secondary to brainstem involvement, and obstructive sleep apnoea due to obesity and muscle dystonia. She was hospitalised for acute respiratory decompensation secondary to hospital-acquired pneumonia. We review the anaesthetic implications of this disease and discuss its impact on preoperative, intraoperative and postoperative management.
\end{abstract}

Keywords: general anaesthesia, Leigh's syndrome, mitochondrial myopathies

\section{INTRODUCTION}

Leigh's syndrome, a subacute necrotising encephalomyelopathy, was first described by Dennis Leigh in 1951.(1) It is a hereditary neurodegenerative disorder that belongs to a family of disorders classified as mitochondrial myopathies. Other mitochondrial myopathies include MELAS (mitochondrial encephalopathy, lactic acidosis and stroke-like episodes) syndrome and NARP (neurogenic muscle weakness, ataxia and retinitis pigmentosa).

Leigh's disease has an estimated prevalence of approximately 1 in 40,000 live births. ${ }^{(2)}$ It presents during infancy with failure to thrive, developmental delay and loss of developmental milestones. Degeneration of the brainstem and basal ganglia occurs early in the course of the disease, and as brainstem function deteriorates, central hypoventilation becomes a key concern. Laboratory investigations of persons with this disease will reveal elevated serum lactic acid and pyruvate levels, and radiological imaging will show symmetrical, bilateral brainstem lesions that generally involve the basal ganglia, most often the putamen. Given the ubiquitous nature of mitochondria in the body and their role in energy production, the disease results in the defective function of several organ systems. Respiratory failure is the common cause of death and postoperative morbidity in patients with Leigh's syndrome. ${ }^{(3)}$

\section{CASE REPORT}

A 17-year-old female patient, who weighed $58 \mathrm{~kg}$, was scheduled for tracheostomy under general anaesthesia. She was diagnosed with Leigh's syndrome at the age of 12 years based on: (a) clinical features such as neuroregression; (b) radiological findings of restricted diffusion in the basal ganglia, in keeping with necrosis; and (c) laboratory findings of elevated serum lactic acid secondary to impaired mitochondrial respiratory chain function, which resulted in metabolic acidosis.

The patient's respiratory complications included muscle dystonia secondary to basal ganglia necrosis and central hypoventilation with obstructive sleep apnoea, for which she was on home bi-level positive airway pressure (BiPAP) ventilation. The patient was dependent on nasogastric tube feeding. Her long-term medications included mitochondrial cofactor thiamine, carnitine (aids in the excretion of harmful metabolites), antioxidant coenzyme Q10, tetrabenazine, levetiracetam and baclofen (to treat generalised dystonia). Within the same month as her current admission, the patient had two prior admissions for unresolved metabolic decompensation secondary to intra-abdominal sepsis. Her current admission was for respiratory decompensation secondary to hospital-acquired pneumonia. The patient was intubated in the emergency department and admitted to the paediatric intensive care unit for ventilatory support. Tracheostomy creation was decided for long-term ventilation and ease of toileting. Preoperative laboratory investigations revealed the following: lactate level $2 \mathrm{mmol} / \mathrm{L}$, arterial blood gas $\mathrm{pH} 7.46, \mathrm{pCO}_{2}$ $40.4 \mathrm{mmHg}, \mathrm{pO}_{2} 122 \mathrm{mmHg}$, standard $\mathrm{HCO}_{3} 28.5 \mathrm{mmol} / \mathrm{L}$, base excess $4.4 \mathrm{mmol} / \mathrm{L}$ and $\mathrm{SaO}_{2} 98.6 \%$.

${ }^{1}$ Department of Anaesthesia, National University Health System, Singapore

Correspondence: Dr Aileen Tan Ling Wan, Resident, Department of Anaesthesia, National University Health System (NUHS), Main Building Level 3, 5 Lower Kent Ridge Road, Singapore 119074. aileen.tan@mohh.com.sg 
To minimise fasting time, the patient was made to fast for only six hours before the surgery. She was then transported to the operating room together with a portable ventilator, which was on synchronised intermittent mandatory ventilatory mode. Preoperatively, the doors of the operating room were left open for as long as possible to allow escape of residual inhalational agents from the previous surgery, and the anaesthetic circuit was flushed to minimise contamination with inhalational agents. The operating theatre was warmed up to maintain an ambient temperature of $23^{\circ} \mathrm{C}$, and a malignant hyperthermia resource kit was brought into the operating theatre so that it would be immediately available in the event it was required. An automated blood pressure monitor, pulse oximeter, electrocardiograph and end-tidal capnograph were used for intraoperative monitoring. The intra-arterial line that was inserted into the right radial artery of the patient during her stay in the intensive care unit was also used for intraoperative monitoring.

Anaesthesia was induced and maintained with $150 \mathrm{mg}$ of intravenous ketamine in divided doses of $50 \mathrm{mg}$. Concurrently, $0.4 \mathrm{mg}$ of glycopyrrolate was given for the copious secretions that built up due to respiratory infection and as a side effect of ketamine. Suctioning of secretions was also performed to minimise obstruction of the airway. To blunt the laryngeal reflexes during airway manipulation, $5 \mathrm{~mL}$ of $1 \%$ lignocaine was given via the intratracheal route. Anaesthesia was also maintained using intravenous remifentanil at a rate of $0.6 \mathrm{mg} / \mathrm{hr}$. A $0.9 \%$ normal saline intravenous drip was used as a maintenance drip throughout the procedure. The patient was ventilated on pressure control mode, with attention given to the maintenance of a low normal end-tidal carbon dioxide of $30-34 \mathrm{mmHg}$. The portable ventilator was used throughout the procedure, eliminating exposure to inhalational agents that may still be present in the anaesthesia circuit. Oxygen saturation was maintained between $97 \%$ and $100 \%$ on fractional inspired oxygen of $60 \%$. Arterial blood gas measured intraoperatively showed $\mathrm{pH}$ at 7.479, $\mathrm{pCO}_{2}$ at $33.4 \mathrm{mmHg}$, $\mathrm{pO}_{2}$ at $147.3 \mathrm{mmHg}$, standard $\mathrm{HCO}_{3}$ at $25.5 \mathrm{mmol} / \mathrm{L}$, a base excess of $0.7 \mathrm{mmol} / \mathrm{L}$ and $\mathrm{SaO}_{2}$ at $98.8 \%$. Lactate and plasma glucose levels were at $2.55 \mathrm{mmol} / \mathrm{L}$ and $4.8 \mathrm{mmol} / \mathrm{L}$, respectively. The procedure lasted for 45 mins.

After the operation, the patient was transferred back to the paediatric intensive care unit. The postoperative course was not complicated, and the patient was weaned off invasive ventilation to BiPAP, and discharged home with it on postoperative Day 15

\section{DISCUSSION}

Respiratory abnormalities are common in patients with Leigh's syndrome due to involvement of the basal ganglia and brainstem. Preoperative evaluation focusing on the respiratory system is essential in identifying comorbid conditions that will impact anaesthetic care. Common problems such as episodes of hypoventilation, irregular respiration and apnoea should be highlighted during anaesthetic evaluation. Obstructive symptoms such as stridor secondary to muscle dystonia may be present. Respiratory infections may complicate lung function and affect ventilatory requirements during general anaesthesia.

Patients with Leigh's syndrome often have lactic acidaemia secondary to impaired mitochondrial function. Impaired glucose regulation secondary to mitochondrial dysfunction may lead to hypoglycaemia during periods of fasting. As such, the duration of fasting should be minimised. Intravenous fluids that contain dextrose should be used to maintain euglycaemia, and solutions containing lactate as a buffer should be avoided. The use of anticonvulsants for seizures and movement disorders is common in patients with Leigh's syndrome. These medications should be continued during the perioperative period. However, it is noteworthy that certain anticonvulsants are hepatic enzyme inducers, which can alter the pharmacokinetics of anaesthetic drugs such as muscle relaxants. ${ }^{(4)}$

Much controversy surrounds the choice of anaesthetic agent used to provide general anaesthesia despite anecdotal reports of their usage without complications. Volatile agents have been associated with the risk of malignant hyperthermia and anaesthesia-induced rhabdomyolysis in myopathic patients. ${ }^{(5)}$ They are also known to depress mitochondrial function. ${ }^{(6)}$ Although malignant hyperthermia has not been reported with Leigh's syndrome, it may be advisable to avoid triggers such as volatile agents, as patients with myopathic conditions may be misdiagnosed with Leigh's syndrome. As barbiturates and propofol are known to depress mitochondrial function, it is important to have a malignant hyperthermia resource kit readily available if they are used. ${ }^{(7,8)}$ Furthermore, propofol is associated with the risk of propofol infusion syndrome, which could worsen the lactic acidosis in patients with Leigh's syndrome. ${ }^{(9)}$ Shenkman et al pointed out that the major anaesthetic problems associated with Leigh's syndrome are the patients' respiratory abnormalities and tendency to develop metabolic acidosis, as well as the possible adverse metabolic effects of the anaesthetic agents. ${ }^{(10)}$

The use of ketamine to anaesthetise a patient with Leigh's syndrome for extracorporeal shock wave lithotripsy was first reported in 1997 by Shenkman et al. ${ }^{(10)}$ In that study, the patient's perioperative course was uneventful, with the patient discharged one day after the operation. In 2004, Shear et al also described its use as an adjunct to spinal anaesthesia, without major complications. ${ }^{(4)}$ These studies guided the use of ketamine as an anaesthetic agent in our patient, in whom we were avoiding the use of volatile agents.

The use of long-acting opioids has been avoided due to their potential for respiratory depression, and there is limited experience with regard to newer opioids such as remifentanil. In a 2004 study by Jacobs et al, (11) the authors reported successful usage of remifentanil for scoliosis surgery in patients with Leigh's syndrome. ${ }^{(11)}$ Remifentanil has a short 
duration of action and therefore a lower tendency to cause postoperative respiratory depression. In the present case, the decision to use remifentanil was due to its organ-independent elimination and minimal residual side-effects.

The choice of muscle relaxant is another important factor in the intraoperative care of a patient. The use of succinylcholine is associated with a potential risk of rhabdomyolysis and anaesthesia-induced rhabdomyolysis in patients with myopathies. ${ }^{(12)}$ However, the use of intermediate-acting, nondepolarising neuromuscular blocking agents such as atracurium and rocuronium is associated with prolonged recovery from neuromuscular blockade. As such, it is recommended that neuromuscular function be monitored if these neuromuscular blocking agents are used, especially when repeated doses are indicated. In the present case, the patient's airway was secured preoperatively. A minimum of $2 \mathrm{mcg} / \mathrm{kg}$ (greater efficiency was achieved with $4 \mathrm{mcg} / \mathrm{kg}$ ) of remifentanil, infused over $90 \mathrm{~s}$, has been used to establish good intubating conditions. ${ }^{(13)}$ This was a consideration in the event that our patient required reintubation. Muscle dystonia may result in impaired upper airway and lower oesophageal sphincter tone, predisposing the patient to reflux and aspiration. In such cases, the use of rapid sequence intubation and pharmacologic prophylaxis with ranitidine or metoclopramide may be considered when securing the airway. ${ }^{(4)}$

Patients with Leigh's syndrome are at risk of metabolic decompensation, thus attention to normoventilation and acid-base status is important. Hypotension, hypoxia and hypovolaemia may exacerbate lactic acidosis and should be avoided as much as possible. ${ }^{(10)}$ Hyperventilation resulting in hypocapnia might inhibit pyruvate carboxylase and worsen lactic acidosis. ${ }^{(14)}$ While the use of a capnograph (for end-tidal carbon dioxide monitoring) and a pulse oximeter is routine in general anaesthesia, the use of invasive monitoring should be guided by the nature of the surgical procedure and patients' comorbid condition. In the present case, given the short duration of the procedure (i.e. 45 mins), no invasive monitor was used except for the intra-arterial line that was inserted in the intensive care unit, prior to the operation. As central regulation of temperature control is impaired in patients with central hypoventilation, ${ }^{(4)}$ attention to temperature management is important. The use of warmers, warmed intravenous fluids and environmental temperature control are essential, and temperature monitoring should be considered for long surgical procedures.

Postoperative respiratory complications have been reported in patients with Leigh's syndrome who underwent general anaesthesia. A previous retrospective study conducted at the Royal Children's Hospital, Melbourne, Australia, on 19 patients with Leigh's syndrome described three patients who developed respiratory failure after general anaesthesia. ${ }^{(15)}$ Although a variety of anaesthetic agents (e.g. halothane and suxamethonium) were used in that study, the same opiate-based premedication (i.e. papaveretum) was used in all patients. The authors of that study concluded that pre-existing respiratory abnormalities (e.g. stridor or irregularity of rhythm) are associated with a significant risk of respiratory failure under general anaesthesia. They also recommend that anaesthesia for routine investigations be avoided under those circumstances. ${ }^{(15)}$

In 2008, Footitt et $\mathrm{al}^{(16)}$ conducted a review of patients with mitochondrial disease who underwent general anaesthesia. In that review, postoperative respiratory failure and metabolic acidosis was reported in a 1-month-old patient with Leigh's syndrome who underwent general anaesthesia with propofol, sevoflurane, fentanyl and midazolam. The patient's clinical deterioration was similar to that in our patient, although no respiratory distress was recorded during our conduct of general anaesthesia. These studies show that close monitoring for respiratory distress, apnoea and irregular respiration is important in postoperative patients with Leigh's syndrome. In the present case, such monitoring was done - our patient was admitted to the intensive care unit postoperatively, and during her stay there, she was weaned to BiPAP.

In patients with Leigh's syndrome who are at a risk of respiratory complications after general anaesthesia, regional anaesthesia is a viable option. Shear et $\mathrm{al}^{(4)}$ reported the uneventful use of spinal anaesthesia for a muscle biopsy conducted on a patient with Leigh's syndrome. The authors also explored the option of regional techniques such as peripheral nerve blockade if the muscle biopsy site was confined to an isolated area. Spinal anaesthesia below the T1 dermatome avoids paralysis of the intercostal musculature and avoids respiratory complications. ${ }^{(4)}$

It is difficult to draw evidence-based conclusions as there are only a handful of reports regarding the anaesthetic care of patients with Leigh's syndrome, given the rarity of the disease. Meticulous individual assessment is thus important. Clinicians should be aware of the greater risk in patients with more severe disease and in those undergoing major surgical procedures. Further prospective work is needed to guide perioperative management and the use of general anaesthetics in patients with Leigh's syndrome. Further studies on the use of newer anaesthetic agents with safer side-effect profiles are also needed to develop evidence-based clinical guidelines.

\section{REFERENCES}

1. Leigh D. Subacute necrotizing encephalomyelopathy in an infant. J Neurol Neurosurg Psychiatry 1951; 14:216-21.

2. Thorburn DR, Rahman S. Mitochondrial DNA-Associated Leigh Syndrome and NARP. In: Pagon RA, Adam MP, Bird TD, et al, eds. GeneReviews ${ }^{\mathrm{TM}}$ [online]. Available at: http://www.ncbi.nlm.nih.gov/books/NBK1173/. Accessed November 29, 2013.

3. Cooper MA, Fox R. Anesthesia for corrective spinal surgery in a patient with Leigh's disease. Anesth Analg 2003; 97:1539-41.

4. Shear T, Tobias JD. Anesthetic implications of Leigh's syndrome. Paediatr Anaesth 2004; 14:792-7.

5. Ragoonanan $\mathrm{V}$, Russell W. Anaesthesia for children with neuromuscular disease. CEACCP 2010; 10:143-7.

6. Bains R, Moe MC, Larsen GA, Berg-Johnsen J, Vinje ML. Volatile 
anaesthetics depolarize neural mitochondria by inhibition of the electron transport chain. Acta Anaesthesiol Scand 2006; 50:572-9.

7. Cohen PJ. Effects of anesthetics on mitochondrial function. Anesthesiology 1973; 39:153-64.

8. Schenkman KA, Tan S. Propofol impairment of mitochondrial respiration in isolated perfused guinea pig hearts determined by reflectance spectroscopy. Crit Care Med 2000; 28:172-7.

9. Bray RJ. Propofol infusion syndrome in children. Paediatr Anaesth 1998; 8:491-9.

10. Shenkman Z, Krichevski I, Elpeleg ON, Joseph A, Kadari A. Anaesthetic management of a patient with Leigh's syndrome. Can J Anaesth 1997; 44:1091-5.

11. Jacobs TF, Plasschaert FS, Bossuyt GP, Szegedi LL, Herregods LL. Anaesthesia for a patient with Leigh's syndrome undergoing surgery for scoliosis. Acta Anaesthesiol Belg 2004; 55:57-9.

12. Larsen UT, Juhl B, Hein-Sörensen O, de Fine Olivarius B. Complications during anaesthesia in patients with Duchenne's muscular dystrophy (a retrospective study) Can J Anaesth 1989; 36:418-22.

13. Erhan E. Ugur G, Alper I, Gunusen I, Ozyar B. Tracheal intubation without muscle relaxants: remifentanil or alfentanil in combination with propofol. Eur J Anaesthesiol 2003; 20:37-43.

14. Ward DS. Anesthesia for a child with Leigh's syndrome. Anesthesiology $1981 ; 55: 80-1$

15. Gratten-Smith PJ, Shield LK, Hopkins IJ, Collins KJ. Acute respiratory failure precipitated by general anesthesia in Leigh's syndrome. J Child Neurol 1990; 5:137-41.

16. Footitt EJ, Sinha MD, Raiman JA, et al. Mitochondrial disorders and general anaesthesia: a case series and review. Br J Anaesth 2008; 100:436-41. 